\title{
Impact of an integrated care programme on patient- reported outcomes for mild to moderate mental health conditions in Singapore: a pilot study
}

\author{
David Choon Liang $\underline{\text { Teo }}^{1, *}$, MRCPsych, FAMS, Shi $\underline{\text { Yan }}^{2, *}$, MD, Michelle Su Qing $\underline{\text { Tan }}^{1}$, MPsych, Irene $\underline{\text { Tirtajana }}^{3}$, MBBS, MMed, \\ Hui Khim Lim ${ }^{2}$, MSocsci, Seyed Ehsan Saffari ${ }^{4}$, PhD, Andrew Lai Huat Peh${ }^{1}$, MMed, FAMS
}

\section{INTRODUCTION}

In recent years, substantial research efforts have focused on reducing the global burden of mental illness. However, translation of research into real-world clinical services has lagged behind. ${ }^{(1)}$ Large gaps in the provision of mental health services persist, even in developed countries. ${ }^{(2-4)}$ Although there has been a burgeoning focus on improving mental health services in Singapore, ${ }^{(5)}$ recent studies have demonstrated a significant treatment gap for mental disorders. ${ }^{(6-8)}$

There is a high prevalence of mental disorders in Singapore. ${ }^{(9,10)}$ However, the 2010 Singapore Mental Health Study found that only $31.7 \%$ of individuals with mental disorders had ever sought help, and only $8.4 \%$ of those were managed by primary care physicians (PCPs). ${ }^{(8)}$ Many mental disorders can be effectively managed in primary care settings, ${ }^{(11)}$ and the integration of mental health services in community settings has been shown to improve patient outcomes. ${ }^{(12)}$ Community mental health services have well-documented advantages, including better accessibility and acceptability of care, and reduced stigma and social discrimination. ${ }^{(13-15)}$ Therefore, a key thrust of the Community Mental Health Masterplan of 2012 was to strengthen primary care to deliver accessible mental health services in the community. ${ }^{(16)}$ Nevertheless, the follow-up Singapore Mental Health Study in 2016 found that the treatment gap for mental disorders remained high at $78.6 \% .{ }^{(7)}$ A local study found that PCPs in Singapore tend to shy away from managing mental conditions because of inadequate support and perceived lack of training. ${ }^{(17)}$ These findings underscore the pressing need to continually enhance primary care mental health services and develop real-world interventions to bridge the treatment gap in Singapore.

Integrated care programmes have been developed in many countries worldwide to support the significant demand for mental health services in primary care settings. ${ }^{(18)}$ In these programmes, patients who are within the patient base of primary care service networks receive integrated mental healthcare with input from specialist mental health professionals within the community. PCPs remain the primary treating physicians and work in close collaboration with specialist mental health professionals to support the mental health needs of their patients.
To enhance the integration of mental health in primary care in Singapore, Changi General Hospital launched the Health Wellness Programme (HWP), a pilot integrated care programme, in 2013. The HWP is a restructured hospital-led, integrated community mental health programme comprising psychiatrists and therapists (clinical psychologists and counsellors). It provides communitybased specialised psychotherapy services to patients with mild to moderate mental health conditions who are managed by PCPs in the eastern region of Singapore. The HWP employs a shared care model that is characterised by close collaboration between members of the team and PCPs. The PCPs receive specialist psychiatric liaison support for patients whom they refer to the programme for psychotherapy. The therapists in the HWP, in discussion with psychiatrists at weekly case conferences, provide regular feedback to the referring PCPs regarding the progress of patients in therapy. PCPs may, in turn, flag patients who are unstable or unresponsive to first-line treatments to attend consultations with the programme's psychiatrists. These patients may be given an early appointment to see a psychiatrist at the specialist outpatient clinic in Changi General Hospital for timely escalation of care.

This stepped care model provides patients in primary care with timely access to specialised psychotherapy interventions within the community, allowing for more effective integration of mental health services within primary care. By empowering and supporting PCPs in managing mental health conditions in the community, our integrated care programme helps to address two key barriers and challenges to mental healthcare in Singapore, namely the stigma associated with seeking tertiary psychiatric treatment and suboptimal accessibility (in terms of cost, scheduling and transport) of mental health services. ${ }^{(7)}$

The primary aim of this study was to examine the impact of the HWP on mental health-related patient-reported outcomes (PROs) for patients enrolled within the programme. The secondary aim was to describe the demographic and clinical profiles of the study participants.

\section{METHODS}

This prospective study involved patients who were referred by PCPs to the HWP for psychotherapy and were successfully enrolled in

${ }^{1}$ Department of Psychological Medicine, Changi General Hospital, ${ }^{2}$ Singapore Health Services, ${ }^{3}$ Department of Psychiatry, Ng Teng Fong General Hospital, ${ }^{4} \mathrm{Centre} \mathrm{for} \mathrm{Quantitative}$ Medicine, Duke-NUS Medical School, Singapore

*These two authors contributed equally as first author in this work.

Correspondence: Dr David Choon Liang Teo, Consultant, Department of Psychological Medicine, Changi General Hospital, 2 Simei Street 3 , Singapore 529889. david.teo.c.l@singhealth.com.sg 
the programme from August 2013 to March 2015. Written informed consent was obtained from all patients. Patients who were included were (a) aged $\geq 21$ years; (b) able to provide informed consent; and (c) had a mild to moderate mental health condition, as defined by a Global Assessment of Functioning (GAF) score of 51-90. Patients with an unstable mental state, delirium or dementia; psychiatric emergencies; suicidal, violent or aggressive behaviours; illicit drug use; personality disorders; and those with a GAF score $\leq 50$ were excluded, as they would be more appropriately managed in specialist settings. This study was exempted from review by the institutional review board, as it was designed to examine the impact of a healthcare service programme.

The participating PCPs referred patients under their care for mental health diagnoses if they met the inclusion criteria of the programme. Thereafter, HWP therapists performed intake assessments to determine the patients' suitability for enrolment into the programme. Subsequently, accepted participants underwent psychotherapy sessions, as indicated. The therapists had the flexibility to choose suitable psychotherapy approaches in collaboration with the patients. The number of treatment sessions was decided based on the therapists' judgement of the patients' clinical needs and agreed goals of treatment. Examples of psychotherapy approaches employed were supportive therapy, psychoeducation, problem-solving therapy, cognitive behavioural therapy, and acceptance and commitment therapy.

Throughout the enrolment process, therapists monitored the progress of the participants and liaised with the referring PCPs in a collaborative approach. Psychiatrists provided management recommendations at weekly case conferences with the therapists. The PCPs could also contact the psychiatrists to discuss cases when required, and escalate cases of patients who were more severely unwell to the specialist clinic at Changi General Hospital, Singapore, within seven working days.

Baseline GAF scores and clinical and demographic data were collected by the therapists during intake assessments. The mental health issues of the patients were categorised according to the records of the referring PCPs. Baseline measures were obtained on three patient-reported outcome measures: the EQvisual analogue scale (EQ-VAS) of the EQ-5D-3L, Patient Health Questionnaire-9 (PHQ-9) and Sheehan Disability Scale (SDS). The EQ-5D-3L is the three-level version of the EQ-5D, a family of instruments measuring self-ratings of general health, and consists of two sections: the EQ-5D three-level descriptive system and the EQ-VAS. Only the EQ-VAS was used in the present study. The EQVAS records the respondent's self-rated generic health status, with an endpoint score of 100 indicating the best health an individual can imagine and 0 indicating the worst. ${ }^{(19)}$ The PHQ-9 is a ninequestion instrument used to screen for the presence and severity of depression in the primary care setting. It measures depressive symptoms by scoring each of the nine DSM-IV (Diagnostic and Statistical Manual of Mental Disorders, Fourth Edition) criteria for depression on a scale of 0 (not at all) to 3 (nearly every day). ${ }^{(20)}$ The SDS is a self-report tool for assessment of functional impairment. This single-dimensional measure of global functional impairment ranges from 0 (unimpaired) to 30 (highly impaired). ${ }^{(21)}$
HWP therapists re-administered these three outcome measures to patients every five sessions and at the completion of therapy. Scores at the initial assessment session were considered as pre-intervention scores, and scores from the most recent readministration before the end of the study period were considered as post-intervention scores.

Descriptive statistical analysis was performed for the demographic and clinical data of all study participants. Continuous variables were presented as mean \pm standard deviation, and categorical variables were presented as frequency and percentage. EQ-VAS, PHQ-9 and SDS scores were compared before and after the intervention using paired $t$-tests. Improvements in mean scores and $95 \%$ confidence intervals were presented. Statistical significance was set at $p<0.05$. Statistical analysis was performed using SAS software version 9.4 for Windows (SAS Institute Inc, Cary, NC, USA).

\section{RESULTS}

A total of 232 patients were referred to the programme and assessed. 229 patients were deemed suitable for enrolment, and 228 of them consented to participate in the programme and data collection.

The demographic features of the participants, including age group, gender and clinical characteristics such as number of diagnoses of common mental health conditions, are summarised in Table I. The majority of the participants were middle-aged Singaporean women with medical comorbidities in addition to their mental health conditions. Among the patients enrolled in our programme, major depressive disorder was the most common mental health condition, followed by anxiety and adjustment disorders. About half of the study participants were on treatment with at least one psychotropic medication; selective serotonin reuptake inhibitors (SSRIs) were the most common drugs $(31.7 \%)$

All three PROs (EQ-VAS, PHQ-9, SDS) showed significant improvement after intervention, as shown in Table II. Participants' mean EQ-VAS scores increased from 61.27 to 78.77 ( $p<0.0001$ ), suggesting a better overall quality of life. Mean PHQ-9 scores decreased from 7.73 to 1.42 , ( $p<0.0001)$, indicating an improvement in depressive symptoms. Mean SDS scores decreased from 10.18 to 2.68 ( $p<0.0001)$, indicating a reduction in functional impairment.

\section{DISCUSSION}

To the best of our knowledge, the present study is the first to examine the impact of an integrated community mental health programme on mental health-related PROs for patients with mild to moderate mental health conditions managed in the primary care setting in Singapore. Our findings suggest that the HWP, a pilot integrated primary care mental health programme, is a feasible intervention that improved mental health-related PROs of patients with mild to moderate mental health conditions managed by PCPs. While the results of the present pilot study are preliminary, they bear important implications for the future planning of community mental health services in Singapore. 
Table I. Demographic and clinical characteristics of study participants $(n=228)$.

\begin{tabular}{|c|c|c|c|}
\hline Variable & No. (\%) & Variable & No. (\%) \\
\hline \multicolumn{2}{|l|}{ Age group (yr) } & \multicolumn{2}{|c|}{ No. of diagnoses of common mental illness* } \\
\hline $18-29$ & $37(16.2)$ & 0 & $10(4.6)$ \\
\hline $30-40$ & $38(16.7)$ & 1 & $165(75.3)$ \\
\hline $41-50$ & $46(20.2)$ & 2 & $41(18.7)$ \\
\hline $51-60$ & $46(20.2)$ & 3 & $3(1.4)$ \\
\hline $61-70$ & $38(16.7)$ & \multicolumn{2}{|l|}{ Mental health condition* } \\
\hline$\geq 71$ & $23(10.1)$ & Depression & $92(41.8)$ \\
\hline \multicolumn{2}{|l|}{ Gender* } & Anxiety & $61(27.7)$ \\
\hline Male & $75(33.0)$ & Insomnia & $28(12.7)$ \\
\hline Female & $152(67.0)$ & Adjustment disorder & $46(20.9)$ \\
\hline \multicolumn{2}{|l|}{ Citizenship status } & Stress & $24(10.9)$ \\
\hline Singaporean & $220(96.5)$ & Grief & $7(3.2)$ \\
\hline Permanent resident & $4(1.8)$ & \multirow{2}{*}{\multicolumn{2}{|c|}{$\begin{array}{l}\text { No. of psychotropic } \\
\text { medications prescribed* }\end{array}$}} \\
\hline Foreigner & $4(1.8)$ & & \\
\hline \multicolumn{2}{|l|}{ Ethnicity } & 0 & $110(48.5)$ \\
\hline Chinese & $179(78.5)$ & 1 & $94(41.4)$ \\
\hline Malay & $29(12.7)$ & 2 & $22(9.7)$ \\
\hline Indian & $7(3.1)$ & 3 & $1(0.4)$ \\
\hline Others & $13(5.7)$ & \multicolumn{2}{|l|}{ Current psychotropic medication* } \\
\hline \multicolumn{2}{|l|}{ Religion* } & Selective serotonin & $72(31.7)$ \\
\hline Christianity/Catholicism & $62(32.3)$ & reuptake inhıbıtor & \\
\hline Buddhism/Taoism & $66(34.4)$ & Hydroxyzine & $18(7.9)$ \\
\hline Islam & $31(16.1)$ & Mirtazapine & $21(9.3)$ \\
\hline Hinduism & $3(1.6)$ & Benzodiazepine & $18(7.9)$ \\
\hline Others & $30(15.6)$ & Other hypnotic/sedative & $5(2.2)$ \\
\hline \multicolumn{2}{|l|}{ Marital status } & Tricyclic antidepressant & $1(0.4)$ \\
\hline Single & $80(35.1)$ & Others & $7(3.1)$ \\
\hline Married & $108(47.4)$ & Presence of medical comorbidities* & $121(54.3)$ \\
\hline Divorced/separated & $26(11.4)$ & \multicolumn{2}{|l|}{ No. of medical comorbidities } \\
\hline Widowed & $14(6.1)$ & 0 & $103(46.2)$ \\
\hline \multicolumn{2}{|l|}{ Education level* } & 1 & $57(25.6)$ \\
\hline No education & & 2 & $29(13.0)$ \\
\hline Primary & $10(0.2)$ & 3 & $29(13.0)$ \\
\hline Secondary & & 4 & $4(1.8)$ \\
\hline Diploma & $10(33.3)$ & 5 & $1(0.4)$ \\
\hline A Levels & $43(20.5)$ & \multicolumn{2}{|l|}{ Medical comorbidities } \\
\hline Degree and above & $10(4.8)$ & Diabetes mellitus & $25(11.2)$ \\
\hline \multirow{2}{*}{\multicolumn{2}{|c|}{ Employment status }} & Hypertension & $62(27.8)$ \\
\hline Unemployed & & Hyperlipidaemia & $66(29.6)$ \\
\hline Employed & $40(17.5)$ & Asthma & $15(6.7)$ \\
\hline & $125(54.8)$ & Cancer & $4(1.8)$ \\
\hline & $27(11.8)$ & Ischaemic heart disease & $7(3.1)$ \\
\hline $\begin{array}{l}\text { Student } \\
\text { Retired }\end{array}$ & $9(3.9)$ & Gastrointestinal & $6(2.7)$ \\
\hline Retired & $27(11.8)$ & Thyroid disorder & $10(4.5)$ \\
\hline \multicolumn{2}{|l|}{ Preferred spoken language } & Others & $28(12.6)$ \\
\hline English & $155(68.0)$ & & \\
\hline Mandarin & $63(27.6)$ & & \\
\hline Malay & $7(3.1)$ & & \\
\hline Others & $3(1.3)$ & & \\
\hline
\end{tabular}

*Percentages were calculated based on available data. 
Table II. Changes in patient-reported outcomes after intervention.

\begin{tabular}{|c|c|c|c|c|}
\hline \multirow{2}{*}{$\begin{array}{l}\text { Outcome } \\
\text { measure }\end{array}$} & \multicolumn{2}{|c|}{ Mean \pm SD } & \multirow{2}{*}{$\begin{array}{l}\text { Mean } \\
\text { change } \\
(95 \% \mathrm{Cl})\end{array}$} & \multirow{2}{*}{ p-value* } \\
\hline & $\begin{array}{l}\text { Pre- } \\
\text { intervention }\end{array}$ & $\begin{array}{l}\text { Post- } \\
\text { intervention }\end{array}$ & & \\
\hline EQ-VAS & $61.27 \pm 12.93$ & $78.77 \pm 10.79$ & $\begin{array}{l}17.5 \\
(12.91,22.09)\end{array}$ & $<0.0001$ \\
\hline PHQ-9 & $7.73 \pm 6.82$ & $1.42 \pm 3.17$ & $\begin{array}{l}-6.31 \\
(-8.07,-4.54)\end{array}$ & $<0.0001$ \\
\hline SDS & $10.18 \pm 6.39$ & $2.68 \pm 5.23$ & $\begin{array}{l}-7.5 \\
(-9.98,-5.02)\end{array}$ & $<0.0001$ \\
\hline
\end{tabular}

*Calculated using paired $t$-test. Cl: confidence interval; EQ-VAS: EQ-visual analogue scale; PHQ-9: Patient Health Questionnaire-9; SD: standard deviation; SDS: Sheehan Disability Scale

It has been well established that the traditional hospitalcentric model of specialist care is insufficient to overcome barriers to care, especially for patients with longer-term disabilities. ${ }^{(22-24)}$ Over the past two decades, several models of a collaborative approach between mental health and primary care providers, commonly termed as integrated care, have been proposed and implemented. ${ }^{(25)}$ Previous studies have demonstrated that integration of mental health services in primary care brings about significant clinical and functional improvements for patients with mental disorders. ${ }^{(25-27)}$ To date, community mental health programmes in Singapore have focused on supporting deinstitutionalisation and step-down care from the hospital to the community, neglecting the treatment of mental health conditions by PCPs in the community from initial detection. ${ }^{(28,29)}$

Our integrated care programme bridges the aforementioned gaps by providing access to timely and affordable specialised psychotherapy services in the community. Patients who are accepted into our programme bypass the need to be referred by their PCP to a psychiatrist in a restructured hospital or a private psychotherapist to access specialised psychotherapy services. This circumvents important barriers to help-seeking for mental disorders, such as stigma in seeing a psychiatrist, scheduling difficulties and cost. Furthermore, the collaborative consultationliaison approach of our programme supports the right-siting of care of mild to moderate mental health conditions in the community from the initial diagnosis, through supporting PCPs with specialist consultation, as required, and timely escalation to specialist care for severely ill patients. These features of our programme may have contributed to the improvements in our patients' PROs.

The clinical characteristics of our sample broadly mirror data from the 2010 Singapore Mental Health Study, which showed that $50.6 \%$ of participants had a comorbid medical illness. ${ }^{(30)}$ In our sample, $54.3 \%$ of the participants had a comorbid medical illness. In Singapore, the majority of patients with chronic medical illnesses are followed up in primary care. As such, our integrated care programme may help to fill an important service gap for patients with comorbid physical and mental illnesses by bringing specialised mental healthcare to the primary care setting to support PCPs in their management efforts.

SSRIs are recommended as the first-line treatment for patients with moderate to severe depressive and anxiety disorders. The prevalence of SSRI use was $31.7 \%$ in our study sample. This suggests that the PCPs participating in our programme were confident in initiating evidence-based pharmacotherapy for depressive and anxiety disorders, which affected two-thirds of our participants. Consultation-liaison support provided by psychiatrists for patients enrolled in our programme and access to community-based specialised psychotherapy services to complement pharmacotherapy may account for the sizeable proportion of patients who were prescribed SSRIs by our partner PCPs. While our sample may not be representative of primary care practice in entire Singapore, the improvements in PROs suggest that this model of care could be feasible for wider implementation.

An important limitation of the present study is the lack of a control group. This means that we could not draw definitive conclusions about the impact of the HWP on mental health PROs in primary care. As this was a programme evaluation health service research study, we also could not determine which specific interventions in our programme contributed to the improvement in PROs. The improvements in EQ-VAS, PHQ-9 and SDS scores could be attributed to co-intervention with psychotropic medications. Future studies employing more sophisticated methodology such as waitlist control or cluster randomisation would generate a higher level of evidence. Another limitation of our study is the relatively short follow-up period, making it unclear whether the positive impact of the HWP is sustainable in the longer term. Nevertheless, our pilot data and results provide preliminary evidence that larger studies with a longer follow-up period can build on. Another limitation of our study is that it did not measure outcomes at the health system level, such as healthcare costs. Integrated care programmes are intrinsically resource intense and should demonstrate cost effectiveness before they are implemented on a large scale. They should lead not only to better clinical outcomes but also health system-level and society-level benefits such as reduced overall healthcare costs. ${ }^{(31)}$ Future research can track patient movement between primary and tertiary care over a period to examine whether the implementation of the HWP is associated with lower healthcare costs.

Future research should also include external validation of similar integrated care programmes in another healthcare cluster at a larger scale. Although our study, along with many other studies, employed protocolised interventions that intend to facilitate the replication or adaptation of similar programmes in other healthcare clusters, ${ }^{(32-36)}$ exact implementation strategies and outcomes in another healthcare cluster will likely depend on the clinical profiles of the local patients, capacity of the healthcare professionals, organisational setup and policy support. Health service research studies employing qualitative and mixed methods approaches are required to determine whether a similar integrated care programme can be adopted in another local healthcare cluster. ${ }^{(37)}$

In conclusion, the results of this pilot study provide preliminary evidence that the HWP, an integrated primary care mental health service, improved PROs for patients with mild to moderate mental health conditions who were managed by PCPs in Singapore. 


\section{REFERENCES}

1. Patel V, Saxena S, Lund C, et al. The Lancet Commission on global mental health and sustainable development. Lancet 2018; 392:1553-98.

2. World Health Organization. Mental Health Atlas 2011. Geneva: WHO, 2011

3. Kessler RC, Demler O, Frank RG, et al. Prevalence and treatment of mental disorders, 1990 to 2003. N Engl J Med 2005; 352:2515-23.

4. Alonso J, Codony M, Kovess V, et al. Population level of unmet need for mental healthcare in Europe. Br J Psychiatry 2007; 190:299-306.

5. Ministry of Health, Singapore. Healthy minds, healthy communities: National Mental Health Blueprint, Singapore 2007-2012. Singapore: Ministry of Health, 2010.

6. Chong SA, Abdin E, Sherbourne C, et al. Treatment gap in common mental disorders: the Singapore perspective. Epidemiol Psychiatr Sci 2012; 21:195-202.

7. Subramaniam M, Abdin E, Vaingankar JA, et al. Minding the treatment gap: results of the Singapore Mental Health Study. Soc Psychiatry Psychiatr Epidemiol 2020; 55:1415-24.

8. Chong SA, Abdin E, Vaingankar JA, Kwok KW, Subramaniam M. Where do people with mental disorders in Singapore go to for help? Ann Acad Med Singap 2012; 41:154-60.

9. Toft T, Fink P, Oernboel E, et al. Mental disorders in primary care: prevalence and co-morbidity among disorders. Results from the Functional Illness in Primary Care (FIP) Study. Psychol Med 2005; 35:1175-84.

10. King M, Nazareth I, Levy G, et al. Prevalence of common mental disorders in general practice attendees across Europe. Br J Psychiatry 2008; 192:362-7.

11. Keynejad RC, Dua T, Barbui C, Thornicroft G. WHO Mental Health Gap Action Programme (mhGAP) Intervention Guide: a systematic review of evidence from low and middle-income countries. Evid Based Ment Health 2018; 21:30-4.

12. Druss BG, von Esenwein SA, Compton MT, et al. A randomized trial of medical care management for community mental health settings: the Primary Care Access, Referral, and Evaluation (PCARE) study. Am J Psychiatry 2010; 167:151-9.

13. Kohrt BA, Asher L, Bhardwaj $A$, et al. The role of communities in mental health care in low- and middle-income countries: a meta-review of components and competencies. Int J Environ Res Public Health 2018; 15:1279.

14. Evans-Lacko S, Corker E, Williams P, Henderson C, Thornicroft G. Effect of the Time to Change anti-stigma campaign on trends in mental-illness-related public stigma among the English population in 2003-13: an analysis of survey data. Lancet Psychiatry 2014; 1:121-8

15. Jorm AF. Mental health literacy: empowering the community to take action for better mental health. Am Psychol 2012; 67:231-43.

16. Ong B. Inaugural Chee Kuan Tsee Lecture: mental health care for the $21 \mathrm{st}$ century. Ann Acad Med Singap 2017; 46:258-62.

17. Vaingankar JA, Fong CW, Kwok KW, et al. Managing patients with mental illness in primary care: apprehensions and views of general practitioners. Singapore Fam Physician 2010; 36:22-5

18. Vogel ME, Kanzler KE, Aikens JE, Goodie JL. Integration of behavioral health and primary care: current knowledge and future directions. J Behav Med 2017 40:69-84

19. Rabin R, de Charro F. EQ-5D: a measure of health status from the EuroQol Group. Ann Med 2001; 33:337-43.

20. Kroenke K, Spitzer RL, Williams JB. The PHQ-9: validity of a brief depression severity measure. J Gen Intern Med 2001; 16:606-13.

21. Leon AC, Olfson M, Portera L, Farber L, Sheehan DV. Assessing psychiatric impairment in primary care with the Sheehan Disability Scale. Int J Psychiatry Med 1997; 27:93-105.

22. Thornicroft G, Tansella M. Components of a modern mental health service: a pragmatic balance of community and hospital care: overview of systematic evidence. Br J Psychiatry 2004; 185:283-90.

23. Thornicroft G, Tansella M. The balanced care model: the case for both hospitaland community-based mental healthcare. Br J Psychiatry 2013; 202:246-8.

24. Thornicroft G, Tansella M, Law A. Steps, challenges and lessons in developing community mental health care. World Psychiatry 2008; 7:87-92.

25. Bryan CJ, Morrow C, Appolonio KK. Impact of behavioral health consultant interventions on patient symptoms and functioning in an integrated family medicine clinic. J Clin Psychol 2009; 65:281-93.

26. Bryan CJ, Corso KA, Corso ML, et al. Therapeutic alliance and change in suicidal ideation during treatment in integrated primary care settings. Arch Suicide Res 2012; 16:316-23.

27. Bryan CJ, Corso ML, Corso KA, et al. Severity of mental health impairment and trajectories of improvement in an integrated primary care clinic. J Consult Clin Psychol 2012; 80:396-403.

28. Lim CG, Koh CW, Lee C, Poon WC. Community psychiatry in Singapore: a pilot assertive community treatment (ACT) programme. Ann Acad Med Singap 2005; 34:100-4.

29. Lum AW, Kwok KW, Chong SA. Providing integrated mental health services in the Singapore primary care setting--the general practitioner psychiatric programme experience. Ann Acad Med Singap 2008; 37:128-31.

30. Chong SA, Abdin E, Nan L, Vaingankar JA, Subramaniam M. Prevalence and impact of mental and physical comorbidity in the adult Singapore population. Ann Acad Med Singap 2012; 41:105-14.

31. Nielsen M, Buelt L, Patel K, Nichols LM. The patient-centered medical home's impact on cost and quality: annual review of evidence 2014-2015. In: The Patient-Centered Primary Care Collaborative. Available at: https://www.pcpcc. org/sites/default/files/resources/The\%20Patient-Centered\%20Medical\%20 Home $\% 27 \mathrm{~s} \% 20$ Impact $\% 20$ on $\% 20$ Cost $\% 20$ and $\% 20$ Quality $\% 2$ C $\% 20$ Annual\%20Review\%20of\%20Evidence\%2C\%202014-2015.pdf. Accessed May 19, 2020.

32. Cigrang JA, Rauch SAM, Mintz J, et al; STRONG STAR Consortium. Treatment of active duty military with PTSD in primary care: a follow-up report. J Anxiety Disord 2015; 36:110-4

33. Goodie JL, Isler WC, Hunter C, Peterson AL. Using behavioral health consultants to treat insomnia in primary care: a clinical case series. J Clin Psychol 2009; 65:294-304.

34. Oxman TE, Hegel MT, Hull JG, Dietrich AJ. Problem-solving treatment and coping styles in primary care for minor depression. J Consult Clin Psychol 2008; 76:933-43.

35. Post EP, Miller MD, Schulberg HC. Using interpersonal psychotherapy (IPT) to treat depression in older primary care patients. Geriatrics 2008; 63:18-28.

36. Strosahl KD, Robinson PJ, Gustavsson T. Brief Interventions for Radical Change: Principles and Practice of Focused Acceptance and Commitment Therapy. Oakland, CA: New Harbinger Publications, 2012.

37. Shepardson RL, Buchholz LJ, Weisberg RB, Funderburk JS. Psychological interventions for anxiety in adult primary care patients: a review and recommendations for future research. J Anxiety Disord 2018; 54:71-86. 